\title{
http://revistainvestigacionacademicasinfrontera.com
}

RECIBIDO EL 23 DE ABRIL DE 2018. DICTAMINADO MEDIANTE ARBITRAJE FAVORABLEMENTE 12 DE MAYO 2018.

\section{Herramientas de control estadístico en las Pymes de Hermosillo, Sonora: limitaciones para su utilización}

\author{
María Magdalena Villa Carbajal ${ }^{1}$, Joel Enrique Espejel Blanco ${ }^{2}$, Alma Brenda Leyva Carreras ${ }^{3}$, \\ Paulina Danae López Ceballos ${ }^{4}$
}

\section{Resumen}

El propósito de esta investigación es identificar las razones por las que las Pymes de Hermosillo, Sonora, no utilizan las Herramientas Básicas de la Calidad para obtener información estadística en el control de sus operaciones para la mejora de sus procesos y productos. El estudio es de tipo no experimental, utilizando una metodología cuantitativa y estadística descriptiva para determinar las frecuencias. La muestra se conformó con 160 Pymes, a las cuales se les aplicó un cuestionario semi-estructurado. Los resultados obtenidos muestran que el grado de conocimiento y utilización de herramientas básicas de la calidad es bajo y para los ejecutivos de las Pymes, la falta de tecnología, de recursos humanos y de recursos financieros afectan en alto grado la utilización de las herramientas básicas de la calidad, como instrumentos para la recolección y organización de información relevante para la toma de decisiones.

Palabras clave: Calidad, Pymes, control estadístico

\footnotetext{
${ }^{1}$ Departamento de Administración, Universidad de Sonora, Blvd. Luis Encinas s/n, Colonia Centro, Hermosillo, Sonora, México.rmvilla@eca.uson.mx

${ }^{2}$ Departamento de Economía, Universidad de Sonora. Blvd. Luis Encinas s/n, Colonia Centro, Hermosillo, Sonora, México. jespejel@pitic.uson.mx

${ }^{3}$ Departamento de Administración, Universidad de Sonora, Blvd. Luis Encinas s/n, Colonia Centro, Hermosillo, Sonora, México. alma brenda_1@hotmail.com

${ }^{4}$ Departamento de Matemáticas, Universidad de Sonora. Blvd. Luis Encinas s/n, Colonia Centro, Hermosillo, Sonora, México.pdanae@mat.uson.mx
} 


\title{
http://revistainvestigacionacademicasinfrontera.com
}

\begin{abstract}
The purpose of this research is to identify the reasons why the SMEs of Hermosillo, Sonora, do not use the Basic Tools of Quality to obtain statistical information in the control of their operations for the improvement of their processes and products. The study is of non-experimental type, using a quantitative methodology and descriptive statistics to determine the frequencies. The sample consisted of 160 SMEs, to which a semi-structured questionnaire was applied. The results obtained show that the level of knowledge and use of the basic quality tools is low and for SME executives, the lack of technology, human resources and financial resources greatly affect the use of the basic tolos of quality, as instruments for the collection and organization of relevant information for decision making.
\end{abstract}

Key words: Quality, SMEs, statistics control

\section{Introducción}

Las pequeñas y medianas empresas (Pymes) han sido objeto de numerosas investigaciones con el propósito de identificar factores que les permitan obtener mejores resultados, a través de la implementación de procesos y técnicas que les permitan desarrollar una gestión más eficiente y ser competitivas en el mercado (Kim, Knotts y Jones, 2008). Las Pymes, al igual que las grandes empresas, deben tener la capacidad para responder oportunamente a los cambios socioeconómicos que implica la economía global y esto requiere tomar decisiones oportunas y eficaces, sustentadas en un soporte metodológico para la mejora e innovación de sus productos y procesos (Ahmedova, 2015; Montilla, 2015), Los nuevos desafíos que enfrentan las organizaciones, dada la internacionalización de la economía, son la competitividad, la productividad y la gestión de procesos, por lo que las Pymes deberán adoptar una orientación mundial, una dinámica acorde a los cambios del entorno, ser más flexibles y apuntar a la mejora continua (Alisic, 2013). 


\section{http://revistainvestigacionacademicasinfrontera.com}

Las Pymes mexicanas proporcionan el $40 \%$ de las fuentes de empleo, con un crecimiento del $81 \%$ en la década de 1999 a 2009, sin embargo su contribución al PIB nacional bajó del 42\% al 34.7\% ENAPROCE (2015). La capacitación de los empleados y el uso de tecnologías de la información son factores que inciden directamente en el desarrollo y crecimiento de las Pymes Ávila (2014), sin embargo, de acuerdo con ENAPROCE (2015), sólo el 30.9\% de la pequeña y el 43.6\% de la mediana empresas en México implementaron instrumentos de mejora continua ante problemas presentados en el proceso de producción en el 2014 y, no obstante que más del 93\% de las Pymes utilizan equipo de cómputo, menos del $28.8 \%$ las utilizan para el control de sus operaciones, esto parece indicar que las Pymes no cuentan con sistemas de control que les proporcionen información pertinente para la toma de decisiones (Velázquez, Cerón y Rodríguez, 2016).

La calidad ha sido adoptada por las empresas como una estrategia, filosofía o un modelo para lograr la mejoría permanente del aspecto organizacional-gerencial y alcanzar los objetivos organizacionales, a través de controles que eviten desvíos en los procesos y productos (Camisón, 2007). Con base en lo anterior, el propósito de esta investigación es identificar las razones por las que las Pymes no utilizan las Herramientas Básicas de la Calidad para obtener información estadística en el control de sus operaciones para la mejora de sus procesos y productos.

\section{Marco Teórico}

La implementación de sistemas de calidad requiere de la utilización de técnicas y herramientas de medida para identificar las causas que provocan desviaciones y aplicar medidas correctivas oportunas y eficientes, en donde todos los integrantes de la organización, incluyendo los niveles operativos, deben participar, a través de una metodología y pueden tener aportaciones significativas en la identificación de problemas, descripción de síntomas, identificación de causas, comprobación de teorías, con recogida y análisis de datos, diseñando, proponiendo e implementando medidas correctivas y estableciendo controles (Gutiérrez, 2014). 


\section{http://revistainvestigacionacademicasinfrontera.com}

Las herramientas de calidad se caracterizan por dar información para identificar áreas problemáticas o de oportunidad y poder enfocar los esfuerzos y los recursos hacia ellas (Hitt, 2015). Las herramientas básicas de la calidad constituyen un grupo de herramientas estadísticas para el control de las operaciones y una de sus características es la libertad en cuanto al grado y orden en su aplicación, así como la amplitud y forma de empleo, en función de sus propósitos, recursos, momento de planeación e implementación, entre otros factores (Clement, 2004). Estas herramientas constituyen un grupo de herramientas sencillas y prácticas que pueden ser utilizadas en los niveles operativos para proporcionar información para la toma de decisiones en los niveles superiores.

Las herramientas que consideraremos en este trabajo son las herramientas básicas de la calidad (HBC), propuestas por Karou Ishikawa (1986), las cuales se caracterizan por dar información estadística para poder observar y seleccionar los problemas y poder actuar de forma que se incremente el grado de acierto en la resolución de problemas, clasificadas por Galgano (1995), en Camisón, Cruz y González (2007) de acuerdo a sus funciones, como se muestra en la Figura 2.1.

\begin{tabular}{|l|l|l|}
\hline \multirow{2}{*}{ Fundamentos } & \multicolumn{1}{|c|}{ funciones } & \multicolumn{1}{c|}{ Herramientas } \\
\hline & Recoger los datos & Hoja de recogida de datos (hoja de verificación \\
\cline { 2 - 3 } & Interpreta los datos & His tograma \\
\hline \multirow{2}{*}{ Pilares } & Estudiar las relaciones causa-efecto & Diagrama de espina \\
\cline { 2 - 3 } & Fijar prioridades & Diagrama de Pareto \\
\hline \multirow{3}{*}{$\begin{array}{c}\text { Instrumentos } \\
\text { auxiliares }\end{array}$} & Estratificación de los datos & Estratificación \\
\cline { 2 - 3 } & Determinar correlaciones & Diagrama de correlación (dispersión) \\
\cline { 2 - 3 } & $\begin{array}{l}\text { Determinar si un proceso es tá bajo } \\
\text { control o no lo está }\end{array}$ & Gráfico de control \\
\hline
\end{tabular}

Figura 2.1.

Las diferentes herramientas de la calidad y sus funciones

Fuente: Galgano (1995, p. 68) en Camisón, Cruz y González (2007, p. 1226) 


\section{http://revistainvestigacionacademicasinfrontera.com}

A nivel internacional, se han hecho estudios relacionados con la calidad, como el de Clemens (2004) cuyos resultados destacan que las empresas de la comunidad Valenciana, que se involucran más en la cultura de la calidad, mediante la utilización de herramientas básicas y administrativas, consiguen mejores resultados en cuanto a reducción de costes y aumento de la productividad, como consecuencia de tener una información de mayor calidad para la toma de decisiones. Lima y Colmenárez (2014) realizaron un estudio en Venezuela, donde encontraron una baja eficiencia en la actuación correctiva en los procesos y esfuerzos significativos, como consecuencia del bajo conocimiento de los beneficios potenciales del uso y aplicación de las herramientas de calidad.

Por su parte, Quispe-Otacoma, Padilla-Martínez, Telot-González y Nogueira-Rivera (2017) realizaron un estudio, en Ambato, Ecuador, sobre las tecnologías de información y comunicación en la gestión empresarial de Pymes comerciales, los resultados muestran que la infraestructura tecnológica de las empresas está limitada al uso de un procesador de textos, hoja de cálculo, software contable e internet, además, no se obtiene información clave de los diferentes procesos, ni cuentan con indicadores para una gestión confiable.

Dentro de la problemática de las pequeñas empresas de los estados de México, Hidalgo, Puebla y Sonora, destaca el uso de las tecnologías de la información, con Hidalgo que presenta un $55 \%$ de empresas que aplican técnicas de calidad, mientras que en Sonora, que cuenta con el mayor porcentaje de empresas certificadas (30\%), sólo el 27\% de sus empresas aplican estas técnicas (Red Pymes-Cumex, 2010).

Ibarra, González, A. y Demuner (2017), realizaron un estudio de la competitividad a nivel micro o empresarial de las Pymes en Baja California. Las 8 dimensiones propuestas para medir la competitividad empresarial presentan bajos niveles, destacando el aseguramiento de la calidad, que incluye el control estadístico como uno de sus principales elementos. Aunque los estudios anteriores no se enfocan al estudio directo de la utilización del control estadístico de la calidad, sí nos han permitido identificar puntos de coincidencia con el presente estudio y llegar a explicar el 


\section{http://revistainvestigacionacademicasinfrontera.com}

análisis en cuestión, ya que fueron realizados a partir de los elementos que conforman las distintas áreas o dimensiones que, al interior de la empresa, contribuyen a su competitividad.

En este trabajo se considera que los tres modelos estratégicos sugeridos por Chafee (1985) y defendidos por Spencer (1994), soportan una propuesta de que la pequeña y mediana empresa pueden iniciar acciones para implementar sistemas de calidad, a partir del modelo mecanicista que requiere la aplicación de herramientas de medición y evaluación en el nivel operativo, pero que, de alguna manera, impactará en la capacidad de los integrantes de la organización para aceptar los cambios y su compromiso hacia la misma (modelo cultural). Sin embargo puede manejarse independientemente de que la empresa esté, en el momento, en condiciones de implementar todo un sistema de gestión que incluya los tres modelos, para poder responder a las necesidades del entorno y alcanzar la competitividad (Camisón, Cruz y González, 2007).

\section{Método}

Se realizó una investigación de tipo exploratorio, no experimental, de diseño transversal, observacional y prospectivo y, con base en las opiniones de los ejecutivos de las Pymes en Hermosillo, Sonora, se obtuvo información válida y suficiente para conocer el grado de conocimiento y utilización de las herramientas básicas de la calidad, así como las razones que dificultan su uso. Las variables estudiadas fueron: "Conoce las Herramientas Básicas de la Calidad", "Utiliza las Herramientas Básicas de la Calidad" y las razones para no utilizar las herramientas consideradas fueron: "Falta de tecnología", "Falta de capacitación del personal" y "Falta de recursos financieros. En la Tabla 3.1. se presenta la ficha técnica de muestreo. La muestra estudiada estuvo conformada por 160 empresas (donde no se incluyeron las seleccionadas para la muestra piloto). 


\section{http://revistainvestigacionacademicasinfrontera.com}

Tabla 3.1.

Ficha técnica de muestreo

\begin{tabular}{ll}
\hline Ámbito geográfico & Hermosillo, Sonora \\
Universo & 520 \\
Unidad muestral & Pymes afiliadas a CANACINTRA Y CANACO \\
Metodología & Encuesta con cuestionario semi-estructurado \\
Procedimiento de muestreo & Muestra finita sin reemplazo \\
Tamaño de la muestra & 160 \\
Error muestral & $\pm .55 \%$ \\
Nivel de confianza & $90 \%, \mathrm{p}=0,5, \mathrm{q}=0.5$ \\
Fecha de trabajo de campo & Septiembre-noviembre de 2016 \\
\hline
\end{tabular}

Para recabar la información, se adecuó el cuestionario utilizado por Clement (2004), para medir la utilización y conocimiento de las herramientas de medición. Para validar el instrumento, se realizó una prueba piloto con 30 empresas de la población objeto de estudio y los resultados obtenidos tuvieron una confiabilidad para Alfa de Cronbach de 0.855; se realizó además, análisis de confiabilidad por dimensión, cuyo resultado se muestra en la Tabla 3.2. para las variables Conoce y Utiliza las Herramientas Básicas de la Calidad.

Tabla 3.2.

Niveles de confiabilidad por dimensión en la prueba piloto de las variables Conoce y Utiliza las Herramientas Básicas de la

Calidad.

\begin{tabular}{lcc}
\hline \multicolumn{1}{c}{ Herramientas } & \multicolumn{2}{c}{ Confiabilidad } \\
\hline & Conoce & Utiliza \\
\cline { 2 - 3 } Diagrama de Pareto & 0.905 & 0.784 \\
Diagrama de Estratificación & 0.921 & 0.862 \\
Hoja de verificación & 0.621 & 0.787 \\
Diagrama de Ishikawa & 0.473 & 0.749 \\
Diagrama de Dispersión & 0.759 & 0.949 \\
Gráfico de Control & 0.575 & 0.564 \\
Histograma & 0.899 & 0.885 \\
\hline
\end{tabular}




\section{http://revistainvestigacionacademicasinfrontera.com}

Después de aplicar el cuestionario, con los datos obtenidos, se utilizó estadística descriptiva para determinar la frecuencia de cada una de las variables.

\section{Resultados}

Los resultados, en general, muestran un porcentaje muy bajo de empresas que conocen las HBC y, de éstas, muy pocas las utilizan. El alto desconocimiento de estas herramientas estadísticas básicas entre los ejecutivos de las Pymes permite suponer que no disponen de información organizada para la toma de decisiones en la mejora de sus operaciones o, aún más, que no cuentan con sistemas formales para programas de mejora. Para los ejecutivos de las Pymes, la falta de tecnología, de recursos humanos y de recursos financieros afectan en alto grado la utilización de las herramientas básicas de calidad, a pesar de que su utilización requiere sólo de un equipo de cómputo y una hoja electrónica, como Exel. Por lo anterior, se puede considerar que la falta de capacitación en el uso de estas herramientas, hace suponer a los ejecutivos que se requiere de mucha tecnología y/o recursos financieros para la integración de estas medidas de control en sus operaciones.

La muestra seleccionada se compone de 160 empresas: 106 pequeñas (66.3\%) y 54 medianas (33.7\%). No se observa diferencia significativa en la composición por giro de las mismas, como se muestra en las Figuras 4.1 y 4.2 . 


\section{http://revistainvestigacionacademicasinfrontera.com}

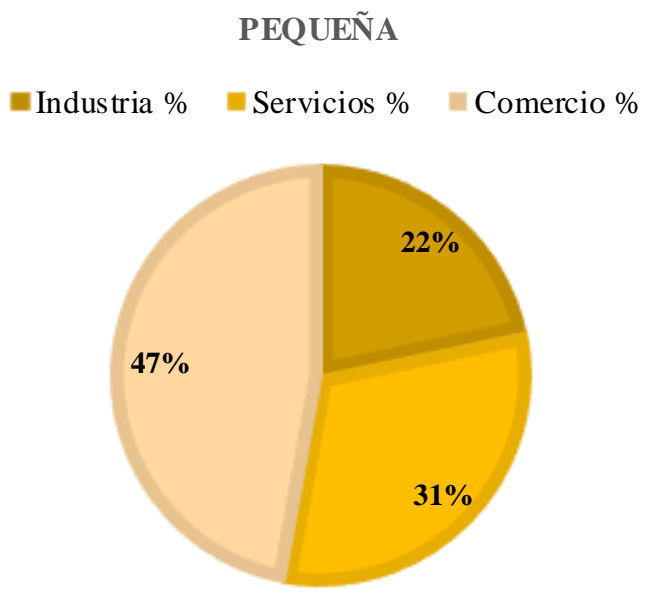

Figura 4.1

Porcentaje de pequeñas empresas, según giro

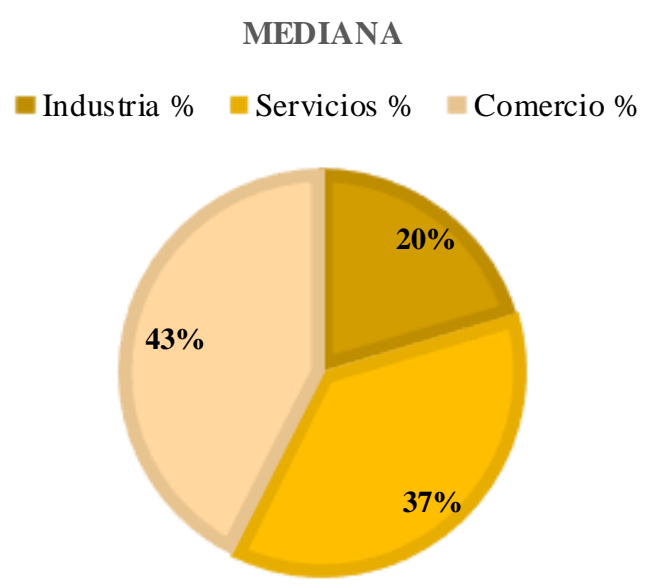

Figura 4.2

Porcentaje de medianas empresas, según giro

El análisis de cada una de las herramientas, por separado, se muestra en las tablas siguientes en orden creciente de acuerdo a las variables conoce y utiliza las Herramientas Básicas de la Calidad.

La Tabla 4.1 muestra que sólo 55 empresas de las 160 encuestadas conocen el Diagrama de Pareto y de estas sólo el 20\% lo utiliza. Se encuentran diferencias significativas no esperadas en los diferentes sectores, ya que los resultados muestran que de las 55 empresas que mencionan conocer el Diagrama de Pareto, el $21.80 \%$ pertenecen al sector industrial, mientras que el $41.80 \%$, corresponde al sector comercio, sector que por el tipo de actividades realizadas no requiere conocer tanto de los controles estadísticos como se esperaría en el sector industrial. El sector servicios destaca en cuanto el grado de utilización de la herramienta, pues el $40 \%$ de los entrevistados que manifestaron conocerla, la utilizan, contra un $13 \%$ del sector comercio y cero por ciento para el sector industrial, es de observar que el sector industrial es el que marca un menor porcentaje en conocer esta HBC y no la utiliza. 


\section{http://revistainvestigacionacademicasinfrontera.com}

Tabla 4.1.

Porcentaje de empresas que Conocen vs Utilizan el Diagrama de Pareto

\begin{tabular}{lcccccccr}
\hline & \multicolumn{2}{c}{ Industria } & \multicolumn{2}{c}{ Servicios } & \multicolumn{2}{c}{ Comercio } & \multicolumn{2}{c}{ Total } \\
Conoce & 12 & $21.80 \%$ & 20 & $36.40 \%$ & 23 & $41.80 \%$ & 55 & $100 \%$ \\
\cline { 2 - 9 } & & & & & & & & \\
Conoce y utiliza & 0 & $0 \%$ & 8 & $40.00 \%$ & 3 & $13.00 \%$ & 11 & $20.00 \%$ \\
Conoce y no utiliza & 12 & $100 \%$ & 12 & $60.00 \%$ & 20 & $87.00 \%$ & 44 & $80.00 \%$ \\
\cline { 2 - 9 } Total: & 12 & $100 \%$ & 20 & $100 \%$ & 23 & $100 \%$ & 55 & $100 \%$ \\
\hline
\end{tabular}

Con un porcentaje ligeramente mayor $21.9 \%$ se encuentra el Diagrama de Ishikawa, Tabla 4.2, para las variables conoce y utiliza las HBC. En esta tabla también se aprecia que el porcentaje más alto en cuanto a conocer la herramienta se encuentra en el sector comercio con un $62.5 \%$, sin embargo para la variable utiliza el sector industrial marcó la tendencia más alta con un 43\%. Es importante destacar que sólo 32 ejecutivos, de los 160 entrevistados, manifestaron conocer esta herramienta que es muy útil para obtener una visión global y estructurada de un problema y permite resumir todas aquellas relaciones entre las causas y efectos de un proceso. Además, de los 32 ejecutivos que conocen la herramienta, menos del $22 \%$ (7) la utilizan. Si consideramos el total de empresas (160) este porcentaje se reduce al $4.37 \%$ de empresas que utilizan el Diagrama de Ishikawa y, siendo esta herramienta de fácil aplicación y que no requiere tecnología especializada, consideramos que no se conoce la importancia y la variedad de sus aplicaciones.

Tabla 4.2.

Porcentaje de empresas que Conocen vs Utilizan el Diagrama de Ishikawa

\begin{tabular}{lcccccccc}
\hline & \multicolumn{2}{c}{ Industria } & \multicolumn{2}{c}{ Servicios } & \multicolumn{2}{c}{ Comercio } & \multicolumn{2}{c}{ Total } \\
Conoce & 7 & $21.88 \%$ & 5 & $15.63 \%$ & 20 & $62.50 \%$ & 32 & $100.00 \%$ \\
\cline { 2 - 10 } & & & & & & & & \\
Conoce y utiliza & 3 & $43 \%$ & 0 & $0.00 \%$ & 4 & $20.00 \%$ & 7 & $21.90 \%$ \\
Conoce y no utiliza & 4 & $57 \%$ & 5 & $100.00 \%$ & 16 & $80.00 \%$ & 25 & $78.10 \%$ \\
\cline { 2 - 10 } Total: & 7 & $100 \%$ & 5 & $100 \%$ & 20 & $100 \%$ & 32 & $100 \%$ \\
\hline
\end{tabular}

Para el Diagrama de Estratificación, el sector industrial muestra el porcentaje más pequeño con menos del $4 \%$ para la variable conoce y no es una herramienta que se utilice en este sector 


\section{http://revistainvestigacionacademicasinfrontera.com}

como se muestra en la Tabla 4.3. Esta herramienta es utilizada mayormente en el sector servicios con un $77.8 \%$ de los que la conocen. De la muestra de 160 empresas, sólo 26 ejecutivos manifestaron conocer esta herramienta y sólo 8 la conocen y la utilizan. Herramienta muy útil para dividir información en base a diferentes factores que son identificados en el momento de obtener los datos e identificar el grado de influencia de determinados factores o variables en el resultado de un proceso y, como su utilización depende de que se utilicen otras herramientas como el Diagrama de Pareto y Dispersión, Gráficos de control o Histogramas, consideramos que es la razón de la poca utilización de esta herramienta, limitando a los ejecutivos de las Pymes para identificar las causas que tienen mayor influencia en la variación de los procesos y descubrir el o los factores más significativos en cuanto a los datos que representan cierto fenómeno, como las diferencias en los diferentes turnos, las líneas, proveedores, clientes, procesos, etc.

Tabla 4.3.

Porcentaje de empresas que Conocen vs Utilizan el Diagrama de Estratificación

\begin{tabular}{lcccccccc}
\hline & \multicolumn{2}{c}{ Industria } & \multicolumn{2}{c}{ Servicios } & \multicolumn{2}{c}{ Comercio } & \multicolumn{2}{c}{ Total } \\
Conoce & 1 & $3.85 \%$ & 9 & $34.62 \%$ & 16 & $61.54 \%$ & 26 & $100 \%$ \\
\cline { 2 - 9 } & & & & & & & & \\
Conoce y utiliza & 0 & $0 \%$ & 7 & $77.80 \%$ & 1 & $6.30 \%$ & 8 & $30.80 \%$ \\
Conoce y no utiliza & 1 & $100 \%$ & 2 & $22.20 \%$ & 15 & $93.80 \%$ & 18 & $69.20 \%$ \\
\cline { 2 - 9 } & 1 & $100 \%$ & 9 & $100 \%$ & 16 & $100 \%$ & 26 & $100 \%$ \\
\hline
\end{tabular}

Sorprenden los resultados del análisis de la herramienta Histograma presentada en la Tabla 4.4., ya que sólo 23 ejecutivos, menos del 15\% del total de la muestra estudiada, manifestaron conocerla y 10 de ellos, menos del 7\% de la muestra, la utiliza. Este dato se destaca, en virtud de que es una herramienta muy conocida, que facilita el reconocimiento y análisis de patrones de comportamiento en la información que no son aparentes a primera vista, además forma parte de los contenidos en los programas de estadística de bachillerato y licenciaturas del área económica administrativa, sin mencionar que es fácilmente graficable en cualquier hoja de cálculo. De los ejecutivos que manifestaron conocer esta herramienta en el sector industrial, el 57\% la utiliza, a 


\section{http://revistainvestigacionacademicasinfrontera.com}

diferencia del sector comercio que, aunque tiene el más alto porcentaje (43.48\%) en el conocimiento de esta herramienta, no la utiliza.

Tabla 4.4.

Porcentaje de empresas que Conocen vs Utilizan el Histograma

\begin{tabular}{lcccccccr}
\hline & \multicolumn{2}{c}{ Industria } & \multicolumn{2}{c}{ Servicios } & \multicolumn{2}{c}{ Comercio } & \multicolumn{2}{c}{ Total } \\
Conoce & 7 & $30.43 \%$ & 6 & $26.09 \%$ & 10 & $43.48 \%$ & 23 & $100 \%$ \\
\cline { 2 - 9 } & & & & & & & & \\
Conoce y utiliza & 4 & $57 \%$ & 6 & $100 \%$ & 0 & $0 \%$ & 10 & $43.50 \%$ \\
Conoce y no utiliza & 3 & $43 \%$ & 0 & $0 \%$ & 10 & $100 \%$ & 13 & $56.50 \%$ \\
\cline { 2 - 9 } Total: & 1 & $100 \%$ & 6 & $100 \%$ & 10 & $100 \%$ & 23 & $100 \%$ \\
\hline
\end{tabular}

Los resultados muestran que el Diagrama de dispersión es conocido por 45, del total de 160 empresas que componen la muestra, porcentaje bajo (28.12\%), sin embargo, muestra un aumento significativo en el porcentaje de ejecutivos que conocen y utilizan esta herramienta 55.6\%. De nuevo los resultados del sector industrial sorprenden con cero conocimiento y utilización de esta herramienta, que es muy útil en las diferentes áreas de las empresas, desde el departamento de producción, hasta ventas o recursos humanos y causa extrañeza los resultados obtenidos en el sector industrial, ya que dentro del sector servicios, de los ejecutivos que conocen esta herramienta casi el 69\% la utiliza y en el sector comercio más del $48 \%$ (Tabla 4.5.).

Tabla 4.5.

Porcentaje de empresas que Conocen vs Utilizan el Diagrama de Dispersión

\begin{tabular}{lcccccccc}
\hline & \multicolumn{2}{c}{ Industria } & \multicolumn{2}{c}{ Servicios } & \multicolumn{2}{c}{ Comercio } & \multicolumn{2}{c}{ Total } \\
Conoce & 0 & $0.00 \%$ & 16 & $35.56 \%$ & 29 & $64.44 \%$ & 45 & $100 \%$ \\
\cline { 2 - 9 } & & & & & & & & \\
Conoce y utiliza & 0 & $0 \%$ & 11 & $68.80 \%$ & 14 & $48.30 \%$ & 25 & $55.60 \%$ \\
Conoce y no utiliza & 0 & $0 \%$ & 5 & $31.20 \%$ & 15 & $51.70 \%$ & 20 & $44.40 \%$ \\
\cline { 2 - 9 } Total: & 0 & $0 \%$ & 16 & $100 \%$ & 29 & $100 \%$ & 45 & $100 \%$ \\
\hline
\end{tabular}

Otro porcentaje sumamente bajo se presentó en la utilización del Gráfico de Control (Tabla 4.6.), herramienta básica para el control y mejoramiento de los productos y procesos, a través del seguimiento de los límites en la variabilidad. Una característica básica de la calidad es el control, 


\section{http://revistainvestigacionacademicasinfrontera.com}

difícilmente las Pymes podrán desarrollar programas de mejora sin la utilización de herramientas que les permitan medir los resultados para detectar y poder analizar eventualidades que signifiquen defectos en el producto o errores en los procesos. De las 160 empresas encuestadas, 24 la conocen y sólo 14 la utilizan, menos del $9 \%$ del total de la muestra.

Tabla 4.6.

Porcentaje de empresas que Conocen vs Utilizan el Gráfico de Control

\begin{tabular}{lcccccccr}
\hline & \multicolumn{2}{c}{ Industria } & \multicolumn{2}{c}{ Servicios } & \multicolumn{2}{c}{ Comercio } & \multicolumn{2}{c}{ Total } \\
Conoce & 3 & $12.50 \%$ & 11 & $45.83 \%$ & 10 & $41.67 \%$ & 24 & $100 \%$ \\
\cline { 2 - 9 } & & & & & & & & \\
Conoce y utiliza & 3 & $100 \%$ & 11 & $100.00 \%$ & 0 & $0.00 \%$ & 14 & $58.30 \%$ \\
Conoce y no utiliza & 0 & $0 \%$ & 0 & $0.00 \%$ & 10 & $100.00 \%$ & 10 & $41.70 \%$ \\
\cline { 2 - 9 } Total: & 3 & $100 \%$ & 11 & $100 \%$ & 10 & $100 \%$ & 24 & $100 \%$ \\
\hline
\end{tabular}

La Hoja de Verificación, con 83 empresas que manifestaron conocerla, muestra el mayor número de empresas que la conocen y utilizan (78.30\%), como se puede observar en la Tabla 4.7. Esto indica que en más del $40 \%$ de las empresas del total de la muestra (160), si se recolectan datos de forma estructurada. La información recogida en una hoja de verificación representa una entrada para el uso de otras herramientas de control de calidad, como el diagrama de Pareto o Dispersión, sin embargo estas herramientas sólo son utilizadas en 11 empresas (tabla 4.1) y 25 (Tabla 4.5), respectivamente, podemos suponer que esta herramienta es utilizada para otros propósitos informativos que no son los del mejoramiento de la calidad.

Tabla 4.7.

Porcentaje de empresas que Conocen vs Utilizan la Hoja de Verificación

\begin{tabular}{lcccccccr}
\hline & \multicolumn{2}{c}{ Industria } & \multicolumn{2}{c}{ Servicios } & \multicolumn{2}{c}{ Comercio } & \multicolumn{2}{c}{ Total } \\
Conoce & 14 & $16.87 \%$ & 33 & $39.76 \%$ & 36 & $43.37 \%$ & 83 & $100 \%$ \\
\cline { 2 - 10 } & & & & & & & & \\
Conoce y utiliza & 10 & $71 \%$ & 33 & $100.00 \%$ & 22 & $61.10 \%$ & 65 & $78.30 \%$ \\
Conoce y no utiliza & 4 & $29 \%$ & 0 & $0.00 \%$ & 14 & $38.90 \%$ & 18 & $21.70 \%$ \\
\cline { 2 - 10 } Total: & 14 & $100 \%$ & 33 & $100 \%$ & 36 & $100 \%$ & 83 & $100 \%$ \\
\hline
\end{tabular}


Núm. 27

ISSN: 2007-8870

\section{http://revistainvestigacionacademicasinfrontera.com}

Los resultados del análisis de los factores que dificultan la utilización de las diferentes herramientas no mostraron diferencias significativas entre la pequeña y mediana empresa, ni entre los diferentes sectores. Las respuestas de los 142 ejecutivos que respondieron esta pregunta, se concentraron en tres factores: falta de tecnología, falta de capacitación de los recursos humanos y falta de recursos financieros.

\section{FALTA DE TECNOLOGÍA}

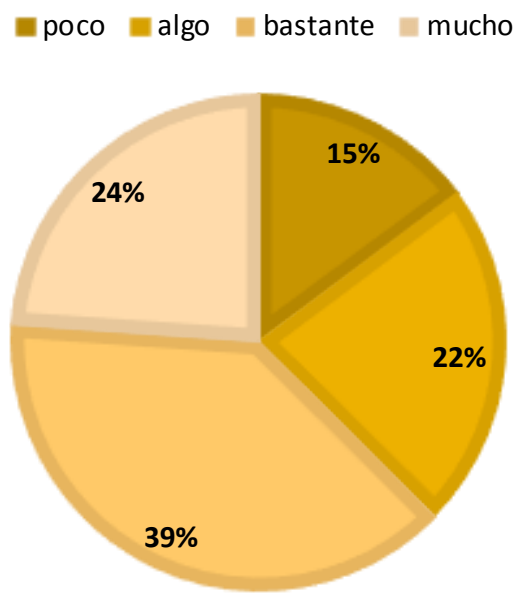

Figura 4.3. Porcentaje de Pymes que no utilizan las herramientas por falta de tecnologìa

A pesar de que sólo 3 empresas manifestaron no contar con equipo de cómputo, casi el $39 \%$ de los ejecutivos consideran que la falta de tecnología afecta bastante y el $24 \%$ mucho la utilización de las diferentes herramientas (Figura 4.3), mientras que el 37\% considera que le afecta poco o algo. 


\section{http://revistainvestigacionacademicasinfrontera.com}

\section{FALTA DE CAPACITACIÒN DEL PERSONAL}

poco $\square$ algo $\square$ bastante $\square$ mucho

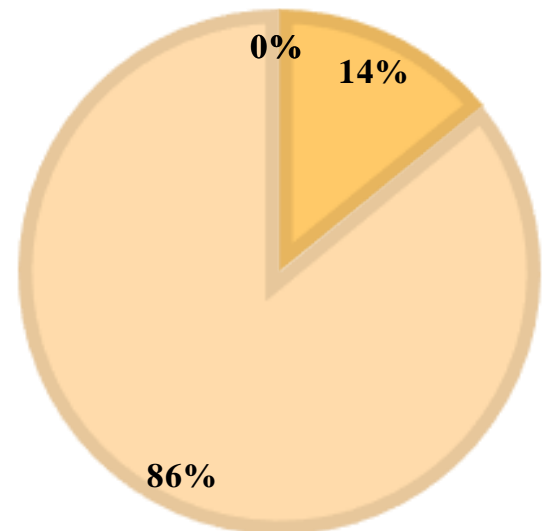

Figura 4.4. Porcentaje de Pymes que no utilizan las herramientas por falta de capacitación del personal

Los resultados del análisis de las variables Falta de Capacitación del Personal y Falta de Recursos Financieros fueron muy contundentes ya que, para la primera (Figura 4.4.), el 14\% de los ejecutivos consideran que la falta de capacitación es un factor que afecta bastante y un $86 \%$ que afecta mucho. Para la segunda, Falta de Recursos Financieros (Figura 4.5.), se obtuvieron porcentajes similares, $26 \%$ para el nivel bastante y $75 \%$ para el nivel mucho. Es importante hacer notar que los ejecutivos están conscientes de la necesidad de capacitación del personal en esta área y de su percepción de que se requieren muchos recursos financieros para utilizar estas herramientas en el mejoramiento de sus procesos. 


\section{http://revistainvestigacionacademicasinfrontera.com}

\section{FALTA DE RECURS OS FINANCIEROS}

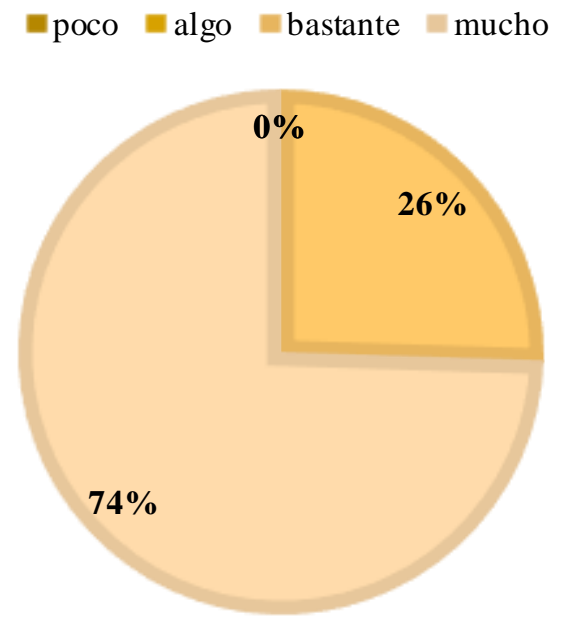

Figura 4.5. Porcentaje de Pymes que no utilizan las herramientas por falta de recursos financieros.

\section{Conclusiones}

El estudio realizado en 160 Pymes de la ciudad de Hermosillo, Sonora, permite concluir que el grado de conocimiento y utilización de herramientas de calidad, como instrumentos para la recolección y organización de información relevante para la toma de decisiones, es bajo. Para los ejecutivos de las Pymes, la falta de tecnología, la falta de recursos humanos y la falta de recursos financieros afectan en alto grado la utilización de las herramientas básicas de la calidad, a pesar de que su utilización no requiere de grandes recursos financieros y pueden implementarse a nivel departamental, incluso personal (Hitt, 2015), esto indica que la percepción de los ejecutivos, en cuanto a que se requiere mucha tecnología y/o recursos financieros para la integración de estas herramientas en el control de sus operaciones, puede deberse a la falta de conocimiento y capacitación en su uso. 


\section{http://revistainvestigacionacademicasinfrontera.com}

Este estudio, aunque discrepa con los resultados obtenidos por Clemens (2004), que obtuvo un grado bastante aceptable en el conocimiento y utilización de las herramientas de calidad, sí coincide con investigaciones realizadas por Red Pymes (2010), Lima y Colmenárez (2014), ENAPROCE (2015), Ibarra, González y Demuner (2017) y Quispe-Otacoma et al. (2017), que también encontraron bajos niveles en la utilización de herramientas de control estadístico. Además, el alto desconocimiento de estas herramientas estadísticas básicas entre los ejecutivos de las Pymes permite suponer que no disponen de información organizada para la toma de decisiones en la mejora de sus operaciones o, aún más, que no cuentan con sistemas formales para programas de mejora.

Como resultado de esta investigación se considera conveniente ahondar en investigaciones que proporcionen información sobre el grado en que las empresas utilizan estas herramientas y que permitan identificar problemas específicos que puedan resolverse con mayor eficiencia, a través del uso de las herramientas básicas de control de la calidad. Se recomienda propiciar el uso de las herramientas básicas de la calidad en las Pymes, mediante una mayor vinculación de las instituciones de Educación superior y estas empresas; revisar los contenidos de los planes y programas de estudio, principalmente de las carreras que estén relacionadas con las áreas económico administrativas y analizar en qué grado y cuáles son las estrategias de enseñanza y aprendizaje adecuadas para que el egresado pueda, no sólo conocerlas, sino aplicarlas cuando se encuentre en el campo laboral.

Con la finalidad de llegar a la implementación de herramientas necesarias y útiles para que las Pymes tomen decisiones que fortalezcan su competitividad, se considera necesario el desarrollo de un esquema de intervención de acompañamiento, por parte de las universidades, que les permita desarrollar sistemas de control para la mejora de sus procesos. Las universidades, apoyadas en la docencia y la investigación para el diseño y desarrollo de proyectos que articulen las experiencias y saberes de la academia con los saberes y experiencias de las Pymes, tendrán la oportunidad de 


\section{http://revistainvestigacionacademicasinfrontera.com}

cumplir con su responsabilidad social hacia el entorno (Martínez de Carrasquero, 2011), en este caso con el sector productivo.

\section{Referencias bibliográficas:}

Ahmedova, S. (2015). Factor for Increasing the Competitiveness of Small and Medium-sized Enterprises (SMEs) in Bulgaria. Procedia. Social and Behavioral Sciences. 195, pp. 11041112 .

Ávila, E. (2014) Las PYMES en México: desarrollo y competitividad. Observatorio de la Economía Latinoamericana. No. 201. En línea: http://www.eumed.net/cursecon/ecolat/mx/2014/cooperacion.html

Alisic (2013). El futuro de la ISO 9001. XV Congreso Internacional de Calidad para Micro, Pequeña y Mediana Empresa. Innovación y Productividad en la Cadena de Valor. México.

Camisón, C., Cruz, S., González T., (2007). Gestión de la Calidad. Conceptos, enfoques, modelos y sistemas. España: Pearson Educación, S.A.

Clement, S. (2004). Utilización y conocimiento de las herramientas de medición y su relación con los costes de calidad en las empresas certificadas en la norma ISO 9000 de la Comunidad Valenciana. Estudios de Economía Aplicada, Vol. 22, núm. 2, pp. 1-20. Asociación Internacional de Economía Aplicada, Valladolid, España.

Chaffee, E. (1985). Three Models of Estrategy. Academy of Management Review, 10(1), p. 8998.

Gutiérrez, H. (2014). Calidad y productividad. Mc Graw Hill: México.

ENAPROCE (2015). Encuesta Nacional sobre Productividad y Competitividad de las Micro, Pequeñas y Medianas Empresas (ENAPROCE). México.

Hitt, M., Ireland, R. y Hoskisson, R. (2015). Administración Estratégica. Competitividad y globalización: conceptos y casos (11va. Edición). México: Cengage Learning 


\section{http://revistainvestigacionacademicasinfrontera.com}

Ibarra, M., González, L., Demuner, M. (2017). Competitividad empresarial de las pequeñas y medianas empresas manufactureras de Baja California. Estudios Fronterizos, 18(35), pp. 107-130.

Ishikawa, K. (1986). ¿Qué es el control total de calidad? La modalidad japonesa. Bogotá: Editorial Norma

Kim, K.; Knotts, T. y Jones S. (2008). Characterizing viability of small Manufacturing enterprises (SME) in the market. Expert Systems with applications. 34, pp. 128-134.

Lima, J. y Colmenárez, M. (2014). Gestión de Calidad y toma de decisiones en Pymes del sector de medios impresos regionales del Estado Lara, Venezuela. Compendium. 32 /27-53.

Martínez de Carrasquero, C. (2011). Responsabilidad Social Universitaria y su Articulación con las Funciones Docencia-Investigación-Extensión para su Vinculación con el Entorno Social. Boletín IESALC Informa de Educación Superior. No. 214. UNESCO.

Montilla, A. (2015). Importancia para las Pymes Venezolanas del uso de los sistemas de soporte a la toma de decisiones. Negotium. Núm. 31 (año 11), pp. 91-111.

Quispe-Otacoma, A., Padilla-Martínez, M., Telot-González, J. y Nogueira-Rivera, D. (2017).

Tecnologías de información y comunicación en la gestión empresarial de pymes comerciales. Ingeniería Industrial, Vol. 38(1), pp. 81-92.

Red Pymes-Cumex (2010). Un estudio comparativo del perfil financiero y administrativo de las pequeñas empresas en México: entidades del Estado de México, Hidalgo, Puebla, Sonora y Tamaulipas. Revista del Centro de Investigación Universidad La Salle, Vol. 9, Núm. 33, pp. 5-30.

Spencer, B. (1994). Models of Organization And Total Quality Management: A Comparison and Critical Evaluation. Academy of Management Review, 19(3), pp. 446-471.

Velázquez, G., Cerón, I. y Rodríguez, C. (2016). Importancia y participación de las Mipymes en la economía mexicana. Recherches en Sciences de Gestión. 114 pp. 45-75. 